Gefässchirurgie $2021 \cdot 26: 402-404$

https://doi.org/10.1007/s00772-021-00801-0

Angenommen: 5. Juli 2021

Online publiziert: 30 . Juli 2021

(c) Der/die Autor(en) 2021

\section{Sterblichkeit nach Anwendung Paclitaxel-beschichteter Devices in der Behandlung der peripheren arteriellen Verschlusskrankheit: Alles nur eine Frage des Geschlechts?}

\section{Christian-Alexander Behrendt}

Forschungsgruppe GermanVasc, Klinik und Poliklinik für Gefäßmedizin, Universitätsklinikum HamburgEppendorf, Hamburg, Deutschland

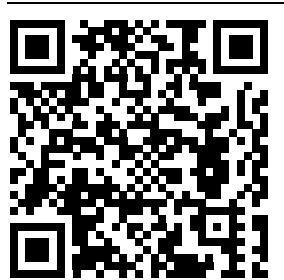

QR-Code scannen \& Beitrag online lesen
Vor mehr als zwei Jahren, im Dezember 2018, löste eine Metaanalyse zu industriegesponserten randomisierten kontrollierten Studien (RCT) eine Welle von Diskussionen aus, die trotz einer gewissen Paclitaxel-Fatique bis heute anhalten [9]. Mit einer zunehmenden Anzahl an Publikationen und Meinungen zum Thema ist es mittlerweile herausfordernd, eine qualifizierte Bewertung der Daten aus Beobachtungsstudien und Metaanalysen vorzunehmen. Letztlich bleibt die Schlussfolgerung daher meist eine subjektive Beurteilung, die persönlicheErfahrungen und andere Erwägungsgründe einschließt. Damit stellt dieses Anwendungsbeispiel evidenzbasierter Medizin (EbM) allerdings keine Ausnahme in der Behandlung der peripheren arteriellen Verschlusskrankheit (PAVK) dar. Ein immanenter Mangel an hochwertiger Evidenz und klaren Leitlinienempfehlungen wurde bereits früher wiederholt hervorgehoben [16].

In den zahlreichen kleineren und größeren Register- und Routinedatenstudien zur Paclitaxel-Debatte konnte bisher ein Sicherheitssignal noch nicht bestätigt werden, während teilweise sogar ein besseres Langzeitüberleben in der PaclitaxelGruppe beobachtet werden konnte $[3,5$, $6,14,15]$. Neuerdings erfreuen sich hierzu auch Sekundäranalysen von RCT-Daten ohne A-priori-Definition der Analysen oder intermediäre Auswertungen zunehmender Beliebtheit, die ebenfalls keine
Assoziation zwischen Paclitaxel-beschichteten Devices und erhöhter Sterblichkeit bestätigen konnten [12].

Auch wenn man die zahlreichen Argumente für oder gegen die Validität, Plausibilität und Relevanz des Sicherheitssignals in der initialen Metaanalyse außer Acht lässt, bleibt letztlich die Frage, ob und warum sich die Kohorten in RCT vs. Beobachtungsstudien diesbezüglich unterscheiden. Anders ausgedrückt: Warum stellen sich vollkommen gegensätzliche Assoziationen in diesen beiden Datenquellen dar? Die hierzu gegründeten Task Forces und Arbeitsgruppen in den Vereinigten Staaten und Europa, unter anderem des Medical Device Epidemiology Network (MDEpiNet), diskutieren bereits seit mehr als einem Jahr, wie ein immanenter Selektionsbias die primären und sekundären Wirksamkeits- und Sicherheitsendpunkte in komplementären Studiendesigns beeinflussen kann.

Gemeinsam mit den internationalen Autoren der einschlägigen Metaanalysen und Beobachtungsstudien zur laufenden Debatte haben wir diese Frage kürzlich eingehender untersucht und sind dabei auf einen interessanten Aspekt gestoßen, der zwar nicht neu ist, aber seit Jahren nur im Ansatz diskutiert wird. Angesichts der zahlreichen Debatten über das Thema "Gender" in Sprache, Gesellschaft und Wissenschaft ist es auffällig, wie wenig konstruktive Diskussionen und insbeson- 


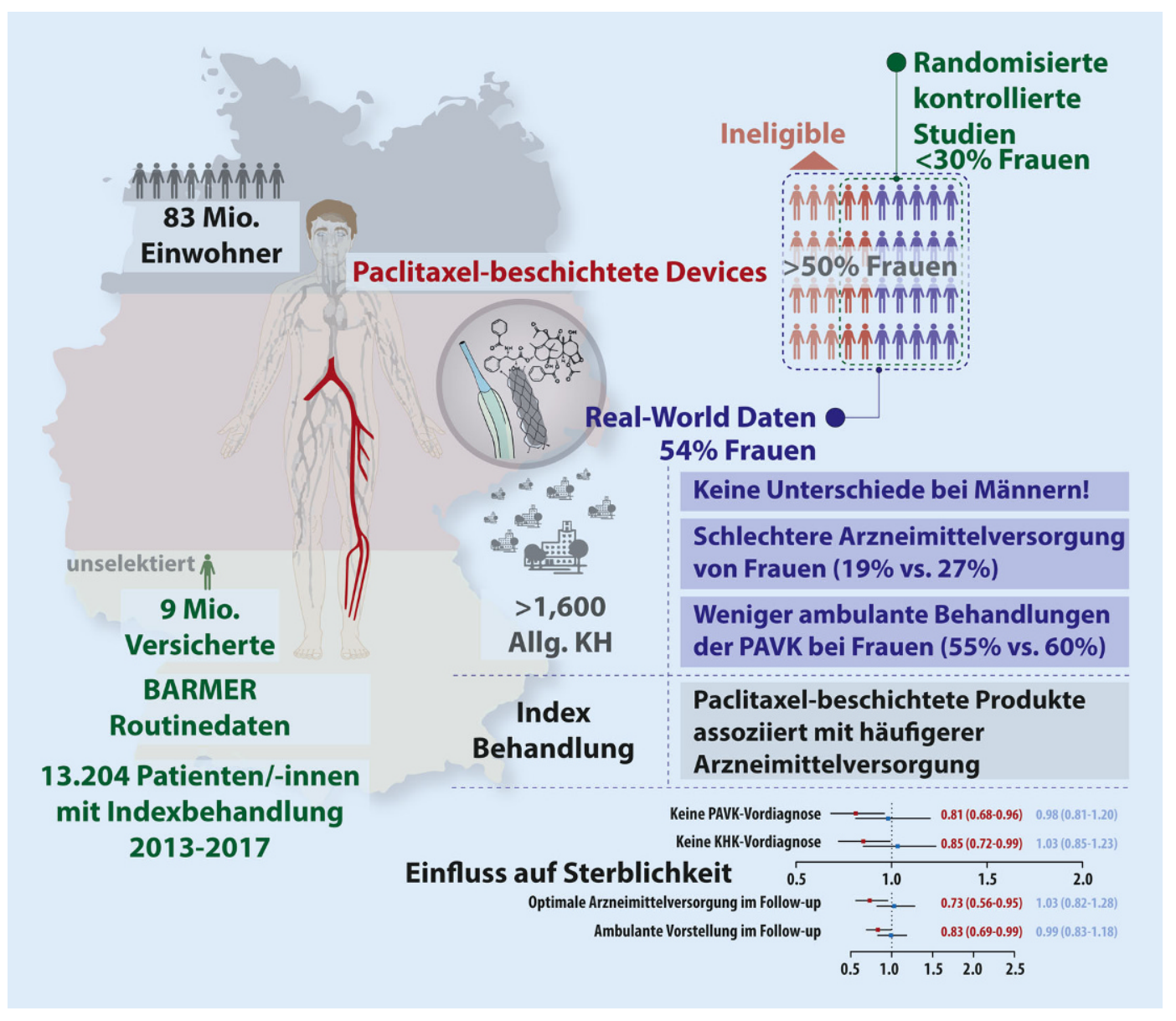

Abb. $1<$ Zentrale Illustration zu den aktuellen Analysen der BARMER Routinedaten, um die Faktoren zu identifizieren, die eine Assoziation zwischen verschiedenen Faktoren und Langzeitsterblichkeit untersucht haben. $K H$ Krankenhäuser, KHK Koronare Herzkrankheit, PAVK Periphere arterielle Verschlusskrankheit dere Veränderungen sich bereits belegen lassen.

Bei einem direkten Vergleich von sogenannten Real-World-Daten und Daten von RCT fällt auf, dass Frauen in rekrutierenden Studien deutlich unterrepräsentiert sind (- Abb. 1). In einer systematischen Analyse von 98 RCT in den Vereinigten Staaten, 69 davon zur PAVK, wurde eine deutliche Unterrepräsentativität von weiblichen $\mathrm{Pa}$ tientinnen nachgewiesen (nur 16\% der PAVK-Studien wiesen eine angemessene Geschlechterratio auf) $[2,8,11]$. Dies bestätigt sich auch bei genauerer Betrachtung der RCT, die in die beiden Metaanalysen der Katsanos-Forschungsgruppe eingegangen waren. Nur etwa $33 \%$ bzw. $29 \%$ Frauen waren dort eingeschlossen worden $[9,10]$, während Frauen in der breiten Versorgungsrealität einen Anteil von bis zu $55 \%$ ausmachen $[4,11]$.

Designbedingte Ausschlüsse von Risikokohorten gehören üblicherweise zu den Charakteristika von RCT und werden seit Jahren als Ursache für verschiedene Selek- tionsbias bzw. die fehlende Generalisierbarkeit der zentralen Schlussfolgerungen diskutiert. Dass besonders gefährdete Subgruppe, z. B. mit erhöhten Blutungsrisiken oder geringer Compliance ausgeschlossen werden, erscheint aus Sicht der Studienplaner nachvollziehbar. Aber warum erfolgt in den RCT eine systematisch geringere Rekrutierung von Frauen? In der internationalen Literatur zeigen sich vor diesem Hintergrund vermehrt Hinweise darauf, dass Frauen bei der komplementären Behandlung der PAVK generell benachteiligt sind. Dies äußert sich z. B. durch eine insgesamt schlechtere Rate an evidenzbasierten Arzneimittelverordnungen und späterer Diagnose sowie Behandlungen der PAVK [4, 13].

In der aktuellen PACLIVASC-Studie (clinicaltrials.gov NCT04683458) wurden nun bundesweit gesammelte Routinedaten der BARMER ausgewertet, um die zugrunde liegenden Faktoren zu identifizieren, die relevante Mortalitätsendpunkte beeinflusst haben [1]. In zahlreichen Sub- gruppen und Interaktionsanalysen stach demnach ein Ergebnis besonders hervor: Während frühere Propensity-Score gematchte BARMER-Analysen einen klaren Überlebensvorteil in der PaclitaxelGruppe nahegelegt hatten [3, 7], konnte in der geschlechterstratifizierten Analyse eine Assoziation zwischen Paclitaxelbeschichteten Devices und besserem Langzeitüberleben nur bei weiblichen Patientinnen nachgewiesen werden, während keine Unterschiede bei Männern auftraten [1].

Diese interessanten Ergebnisse führen zu einer weiteren Hypothese, die auch auf dem kommenden Jahreskongresses der Deutschen Gesellschaft für Gefäßchirurgie und Gefäßmedizin (DGG e.V.) im Rahmen einer internationalen Sitzung zum Paclitaxel-Thema am 15. Oktober 2021 diskutiert wird. Da die Behandlung mit Paclitaxelbeschichteten Devices mit einer höheren Rate an optimalen Arzneimittelverschreibungen, v.a. hinsichtlich Statinen, assoziiert werden konnte, wäre ein besseres 
Langzeitüberleben vor allem in zuvor unterversorgten Patienten/-innen zu erklären. Dies trifft insbesondere auf Frauen zu. Dieser mögliche Behandlungsvorteil wäre in vollständigen bzw. weniger selektierten Real-World-Daten nachweisbar, während der Ausschluss unterversorgter Patienten/-innen in RCT zu einer relevanten Verzerrung führen könnte.

Allerdings würde diese Hypothese nicht ohne gleichzeitige geschlechterspezifische Unterschiede bei der Arzneimitteladhärenz im Langzeitverlauf standhalten. Daher stellt sich für unsere alltägliche Praxis die Frage, ob sich unsere gemeinsamen Bemühungen primär auf unterversorgte weibliche Patientinnen mit einer PAVK oder auf die unzureichende Adhärenz zum Best-Medical-Treatment vor allem männlicher Patienten konzentrieren sollten?

In jedem Fall illustriert dieses Anwendungsbeispiel die wichtige komplementäre Rolle von administrativen und klinischen Registern für Fragestellungen aus dem Bereich der Qualitätssicherung, deren Beantwortung designbedingt nicht mit RCT erfolgen kann.

\section{Korrespondenzadresse}

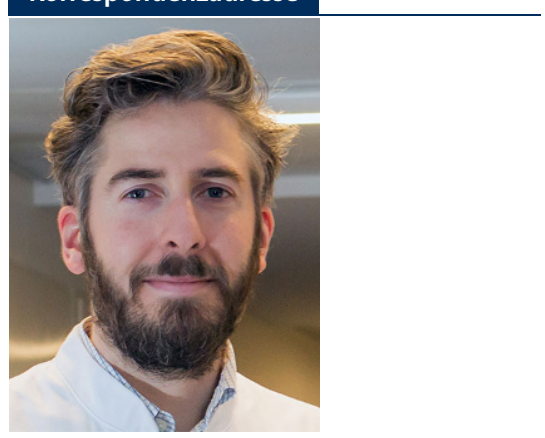

PD Dr. Christian-Alexander Behrendt

Forschungsgruppe GermanVasc, Klinik und Poliklinik für Gefäßmedizin, Universitätsklinikum Hamburg-Eppendorf

Martinistr. 52, 20246 Hamburg, Deutschland behrendt@hamburg.de

Funding. Open Access funding enabled and organized by Projekt DEAL.

\section{Einhaltung ethischer Richtlinien}

Interessenkonflikt. C.-A. Behrendt gibt an, dass kein Interessenkonflikt besteht.

Für diesen Beitrag wurden vom Autor keine Studien an Menschen oder Tieren durchgeführt. Für die aufgeführten Studien gelten die jeweils dort angegebenen ethischen Richtlinien.

Open Access. Dieser Artikel wird unter der Creative Commons Namensnennung 4.0 International Lizenz veröffentlicht, welche die Nutzung, Vervielfältigung, Bearbeitung, Verbreitung und Wiedergabe in jeglichem Medium und Format erlaubt, sofern Sie den/die ursprünglichen Autor(en) und die Quelle ordnungsgemäß nennen, einen Link zur Creative Commons Lizenz beifügen und angeben, ob Änderungen vorgenommen wurden.

Die in diesem Artikel enthaltenen Bilder und sonstiges Drittmaterial unterliegen ebenfalls der genannten Creative Commons Lizenz, sofern sich aus der Abbildungslegende nichts anderes ergibt. Sofern das betreffende Material nicht unter der genannten Creative Commons Lizenz steht und die betreffende Handlung nicht nach gesetzlichen Vorschriften erlaubt ist, ist für die oben aufgeführten Weiterverwendungen des Materials die Einwilligung des jeweiligen Rechteinhabers einzuholen.

Weitere Details zur Lizenz entnehmen Sie bitte der Lizenzinformation auf http://creativecommons.org/ licenses/by/4.0/deed.de.

\section{Literatur}

1. Behrendt C-A, Sedrakyan A, Katsanos K et al (2021) Sex disparities in long-term mortality after paclitaxel exposure in patients with peripheral artery disease: a nationwide claims-based cohort study. JClin Med 10:2978

2. Behrendt CA, Rother U, Rümenapf G et al (2020) Geschlechterspezifische Unterschiede bei der endovaskulären Behandlung der peripheren arteriellen Verschlusskrankheit - Eine systematische Literaturübersicht. Gefässchirurgie 25:587-602

3. Behrendt CA, Sedrakyan A, Peters $F$ et al (2020) Editor's choice-long term survival after femoropopliteal artery revascularisation with paclitaxel coated devices: a propensity score matched cohort analysis. Eur JVasc Endovasc Surg 59:587-596

4. Behrendt CA, Sigvant B, Kuchenbecker Jet al (2020) International variations and gender disparities in the treatment of peripheral arterial occlusive disease-a report from VASCUNET and the International Consortium of Vascular Registries. Eur JVasc Endovasc Surg 60(6):873-880

5. BertgesDJ, Sedrakyan A, Sun Tetal (2020) Mortality after paclitaxel coated balloon angioplasty and stenting of superficial femoral and popliteal artery in the vascular quality initiative. Circ Cardiovasc Interv 13:e8528

6. Böhme T, Noory E, Beschorner U et al (2021) Evaluation of mortality following paclitaxel drug-coated balloon angioplasty of femoropopliteal lesions in patients with ulcerations and gangrene-a single center experience. Vasa 50(2):132-138

7. Heidemann F, Peters F, Kuchenbecker J et al (2020) Long term outcomes after revascularisations below the knee with paclitaxel coated devices: a propensity score matched cohort analysis. Eur $J$ Vasc Endovasc Surg 60(4):549-558

8. Jelani QU, Petrov M, Martinez SC et al (2018) Peripheral arterial disease in women: an overview of risk factor profile, clinical features, and outcomes. Curr Atheroscler Rep 20:40

9. Katsanos K, Spiliopoulos S, Kitrou Petal (2018) Risk of death following application of paclitaxel-coated balloons and stents in the femoropopliteal artery of the leg: a systematic review and meta-analysis of randomized controlled trials. J Am Heart Assoc 7:e11245

10. Katsanos K, Spiliopoulos S, Kitrou Pet al (2020) Risk of death and amputation with use of paclitaxelcoated balloons in the infrapopliteal arteries for treatment of critical limb ischemia: a systematic review and meta-analysis of randomized controlled trials. JVasc Interv Radiol 31:202-212

11. Mayor J, Preventza O, Mills JL Sr et al (2021) Persistent underrepresentation of female patients in US trials of common vascular diseases since 2008. J Vasc Surg 73:e23

12. Nordanstig J, James $S$, Andersson $M$ et al (2020) Mortality with paclitaxel-coated devices in peripheral artery disease. $\mathrm{N}$ Engl J Med 383:2538-2546

13. Peters F, Kreutzburg T, Riess HC et al (2020) Editor's choice-optimal pharmacological treatment of symptomatic peripheral arterial occlusive disease and evidence of female patient disadvantage: an analysis of health insurance claims data. Eur J Vasc Endovasc Surg 60:421-429

14. Saratzis A, Lea T, Yap T et al (2020) Paclitaxel and mortality following peripheral angioplasty: an adjusted and case matched multicentre analysis. Eur JVasc Endovasc Surg 60:220-229

15. Secemsky EA, Kundi H, Weinberg I et al (2019) Association of survival with femoropopliteal artery revascularization with drug-coated devices. JAMA Cardiol 4:332-340

16. Torsello G (2015) Die Gefäßmedizin braucht mehr Evidenz. Gefasschirurgie 20:6-7 